\title{
Pulling together: Tissue-generated forces that drive lumen morphogenesis
}

\author{
Adam Navis ${ }^{1}$ and Celeste M. Nelson ${ }^{1,2^{*}}$ \\ Departments of ${ }^{1}$ Chemical \& Biological Engineering and ${ }^{2}$ Molecular Biology \\ Princeton University, Princeton, NJ 08544
}

(*) Address correspondence to C.M.N.

303 Hoyt Laboratory

William Street

Princeton, NJ 08544

Tel: $609-258-8851$

Fax: 609-258-1247

E-mail: celesten@princeton.edu

Keywords: Mechanical stress; morphodynamics; branching

Abbreviations: CFTR, cystic fibrosis transmembrane conductance regulator; ECM, extracellular matrix; FRET, Förster resonance energy transfer; JNK, c-Jun N-terminal kinase; PCP, planar cell polarity; ROCK, Rho-associated protein kinase; VEGF, vascular endothelial growth factor 


\begin{abstract}
Mechanical interactions are essential for bending and shaping tissues during morphogenesis. A central feature of nearly all internal organs is the formation of a tubular network consisting of an epithelium that surrounds a central lumen. Lumen formation during organogenesis requires precisely coordinated mechanical and biochemical interactions. Whereas many genetic regulators of lumen formation have been identified, relatively little is known about the mechanical cues that drive lumen morphogenesis. Lumens can be shaped by a variety of physical behaviors including wrapping a sheet of cells around a hollow core, rearranging cells to expose a lumenal cavity, or elongating a tube via cell migration, though many of the details underlying these movements remain poorly understood. It is essential to define how forces generated by individual cells cooperate to produce the tissue-level forces that drive organogenesis. The transduction of mechanical forces relies on several conserved processes including the contraction of cytoskeletal networks or expansion of lumens through increased fluid pressure. The morphogenetic events that drive lumen formation serve as a model for similar mechanical processes occurring throughout development. To understand how lumenal networks arise, it will be essential to investigate how biochemical and mechanical processes integrate to generate complex structures from comparatively simple interactions.
\end{abstract}




\section{Introduction}

Many organs are characterized by the presence of a tubular architecture, usually consisting of a central lumen surrounded by epithelial cells. These can be simple straight tubes, as exemplified by the intestine or neural tube, or they may have an intricate branching pattern, observed in the lung, vasculature, and many secretory organs. Lumen formation requires several morphogenetic movements and is governed by the coordinated efforts of genetic and physical mechanisms. Classically, lumens may form by any of several conserved processes. An epithelial sheet may wrap into a cylinder to enclose a lumen. Alternatively, lumens may arise from within a rod of cells, which undergo cellular rearrangements to generate a central cavity during cord hollowing. Lumens may also form by hollowing through a single cell. Once formed, hollow epithelia can undergo branching morphogenesis to extend a lumen in new directions [1-3].

Lumen formation is essential for organogenesis and can serve as a model for fundamental processes that shape development. The morphogenesis of many organs is guided by interactions between physical processes and biochemical signals. Whereas the effects of several genetic and signaling processes have been examined extensively, the mechanical forces that drive morphogenesis remain far less well characterized. Changes in cell shape and tension can have dramatic effects on the architecture and migration of the tissues in which they reside. Cells are capable of not only generating mechanical force, but also of sensing and transmitting forces. Understanding how cells generate and interpret mechanical forces will be essential for understanding morphogenesis. Here we review the physical processes that shape epithelia and how these mechanisms contribute to lumen formation and organogenesis. 


\section{Types of morphogenetic movements}

\subsection{Epithelial bending}

One fundamental mechanical process that occurs during lumen morphogenesis is bending of epithelia. Epithelial bending can initiate new lumens by inducing the invagination of a sheet of cells and can extend new branches by generating deformations that extend an existing lumen in new directions. This process was initially characterized during the development of the chicken neural tube, where tissue deformations are driven by changes in the shape of the neuroepithelial cells, which were found to contract at their apical surfaces [4] (Figure 1A,B). The link between apical constriction of individual cells and bending of epithelial tissue has been best characterized during gastrulation in several species [5-7], which has served as a model for other morphogenetic events. During gastrulation, this change in cell shape bends the embryo and drives involution necessary for differentiating cell types in the early embryo.

Apical constriction can also drive the initial stages of lumen morphogenesis. The vertebrate lung uses recursive branching events to generate a complex and nearly stereotypic airway tree. In the chicken lung, new branches are initiated by localized apical constriction of airway epithelial cells [8] (Figure 1A). Similar changes in cell shape have been suggested to drive branching in the mouse lung [9]. Apical constriction can also generate a new lumenal organ from an epithelial sheet. For example, the Drosophila salivary gland arises from a pit of apically constricting epithelial cells [10], and the resulting invagination initiates the formation of a new lumen. Simple changes in cell shape can drive dramatic bends and folds in epithelia throughout organogenesis. 
Interestingly, recent work suggests that apoptotic cells may generate transient pulling forces that bend the apical surfaces of epithelia. In the Drosophila leg disk epithelium, apoptotic cells help initiate epithelial bending [11]. This transient force precedes the onset of apical constriction, suggesting a mechanical regulation of apical constriction. Similarly, apoptotic cells have been noted at critical locations during bending of the vertebrate neural tube [12]. It will be interesting to determine whether the forces generated by apoptosis serve as a more widespread mechanism for bending epithelia and resolving lumens. Regardless, forces generated from within cells can exert dramatic effects on the surrounding tissue, capable of bending epithelia and initiating new lumen outgrowth.

\subsection{Collective migration}

Once initiated, lumen outgrowth requires epithelial extension, which can be driven by collective migration. As they migrate, epithelial cells maintain adhesive connections to their neighbors while being guided by a group of tip cells at the leading edge. The fruit fly has been instrumental for identifying and characterizing genetic regulators of the physical processes that underlie collective migration during lumenal morphogenesis. In the highly branched tracheal system, which transfers gases throughout the body of the fly, new branches arise by collective migration of cells that enclose a central lumen (Figure 1C). The position of these branches is directed by fibroblast growth factor, which stimulates a group of tip cells to migrate toward the signal and elaborate the network [13-15].

Collective migration is also essential for morphogenesis of many vertebrate organs. Similar to the Drosophila trachea, collective migration of endothelial cells in the vertebrate 
vasculature generates a network that extends throughout the animal (Figure 1C). Vascular development has been examined extensively in zebrafish, where the optical transparency of the developing larva has permitted direct observation of vascular migration in response to a variety of signals. Secretion of vascular endothelial growth factor (VEGF), in particular, plays a key role in the morphogenesis of vertebrate vasculature $[16,17]$. Tip cells at the leading edge of a sprout lead the collective migration of new vascular branches towards the VEGF source. Similarly, semaphorin and plexin signaling can direct the growth and movement of new vessels by guiding cellular migration [18]. Collective migration can also be influenced by mechanical cues. In the zebrafish pronephric duct, fluid flow stimulates collective migration of kidney epithelial cells [19]. Obstructing the ductal lumen, which blocks fluid flow, inhibits cell migration and disrupts kidney morphogenesis.

Collective migration has been well studied during development of the mouse mammary gland. Similar to other lumenal networks, the mouse mammary gland branches through collective migration of groups of cells away from the central lumen [20]. During migration of the mammary gland epithelium, individual cells maintain limited junctional contacts and can be observed migrating within the epithelium [21]. Normal collective migration of these cells depends on contacts with the extracellular matrix (ECM). Changes in the basement membrane or deletion of adhesion proteins can drive increased collective migration from the mammary epithelium [22]. Adhesions transmit mechanical forces during collective migration and allow the epithelium to migrate as a unit, even when led by relatively few, distant cells. 


\subsection{Extending tubes by intercalation and convergent extension}

Tubes may also elongate through individual cellular rearrangements and convergent extension of epithelial cells. Convergent extension is characterized by movement or convergence of cells toward each other in one direction, which drives elongation of the tissue in the perpendicular direction (Figure 1D). Genetic regulators of this process have been well characterized in the Drosophila germ band, an epithelial sheet that dramatically extends the body plan early in fly development [23]. Cells in a sheet like the Drosophila germ band may be polarized in the plane of the epithelium, leading to differences in protein composition of the cells nearest one edge of the sheet. Planar polarization in the germ band generates stripes of actin contractility, which allows cells to coordinate shortening along one axis to facilitate cellular rearrangements to exchange neighbors and elongate the sheet $[24,25]$. To rearrange themselves, cells must also reorganize their cell-cell contacts. During convergent extension in the germ band, this extensive remodeling of adhesive contacts facilitates the rearrangement of neighboring cells [26]. Convergent extension is also a key component of Drosophila hindgut development. As the hindgut develops, it must undergo substantial elongation, which is driven in part by convergent extension of the epithelium. The movements of these cells are patterned by a network of transcription factors that help coordinate hindgut elongation [27].

Convergent extension is also crucial during early vertebrate morphogenesis. In the zebrafish and frog neural tube, planar polarized signals establish the sites of the cellular intercalations that drive convergent extension [28]. Elongation of the Xenopus kidney tube is also driven by convergent extension. In a process that mirrors the cellular movements first observed in Drosophila, planar cell polarity (PCP) establishes bands of actin contractility that drive 
neighbor exchange and help extend the tube [29]. Similarly, myosin IIB drives actin contractility during Xenopus gastrulation that facilitates cellular rearrangements and neighbor exchange, hallmarks of convergent extension [30]. This process is conserved in mammals, where PCP is essential for establishing the domains in which convergent extension also drives tube elongation [31]. Convergent extension has also been proposed to generate the forces that lead to tissue bending in the chick neural tube. Mediolateral bands of actin contractility facilitate migration of cells to promote the bending of the neural tube [32]. Together, these rearrangements of individual cells coordinate to drive epithelial tissue elongation essential for lumen growth in many organs.

\subsection{Cell sorting}

The segregation of cells that will contribute to lumen formation is a fundamental morphogenetic process essential for organogenesis. Cell sorting was initially proposed to be driven by the relative adhesion strength between different cell types [33,34], a property that is regulated by the levels of cadherin expression. Consistently, cells expressing higher levels of cadherins tend to cluster in the center of the population $[35,36]$. Whereas cadherin expression is well correlated with sorting, cadherin engagement can also increase the tension of the cortical actin cytoskeleton. Accordingly, cortical tension, which modulates cell stiffness, has also been shown to drive cell sorting [37, 38]. Cells with higher levels of cortical tension tend to sort to the center of cell aggregates. To determine which of these physical processes drives differential cell sorting, the role of adhesion versus cortical tension was dissected by removing the connection between cadherins and the actin cytoskeleton. In these experiments, cortical tension determined the positioning of cells independently of the cadherin expression levels, highlighting the crucial 
role of actomyosin cytoskeletal tension during cell sorting [39] (Figure 1E). In the early mammalian embryo, cells undergo compaction, which is essential for organizing the early blastocyst lineages. Recent work has demonstrated that the compaction of these cells is driven primarily be pulsatile actomyosin contractility [40]. Cortical tension may represent a common mechanism for organizing cell types in a variety of tissues.

Curiously, simple cell-sorting experiments in culture often lead to different orientations of cells than are observed in the embryo. This is likely due to adhesive interactions between cells and their surrounding microenvironment. In culture, mouse mammary epithelial cells can form a central lumen, however in the absence of proper sorting, lumen formation fails to occur. Here, cell-ECM adhesion can overcome the cell-cell interactions and direct the positioning of individual tissue layers essential for lumen formation [41] (Figure 1E). Whereas interfacial tension can separate tissue layers, interaction with the microenvironment helps guide their proper orientation. Thus, physical cues are essential for directing morphogenesis of organs and the lumens they contain.

\section{Cell biology of force generation and transmission}

\subsection{Actomyosin contractility}

A key driver of mechanical processes throughout morphogenesis is contractility of the actin network generated by myosin activity (Figure 2A). Tension of the actin cytoskeletal network is essential for processes ranging from apical constriction to cell sorting. The effects of actomyosin tension can be clearly observed during dorsal closure in the Drosophila embryo, where actomyosin contractility pulls opposing epithelia together in preparation for their fusion. 
Disrupting the actin cables at the leading edge of these converging epithelial sheets leads to a dramatic recoil, indicative of the strong contractile forces at work in the tissue [42, 43]. Actomyosin contractility in these contexts is a dynamic process. During Drosophila gastrulation, actomyosin contractility is observed as cyclic pulses, suggesting that a ratchet-like mechanism underlies cell and tissue deformations [44].

Polarized actomyosin contractility at the apical surface can generate forces that constrict the lumenal surface of a cell and generate a pyramidal geometry. Together, apically constricting cells can generate dramatic deformations in an epithelial tissue. As described above, apical constriction is essential for many examples of lumen morphogenesis. In the Drosophila salivary gland, apical constriction initiates invagination of an epithelial sheet to begin the formation of the lumen [10]. Apical constriction is also essential during vertebrate morphogenesis. In the developing chicken lung, actomyosin tension at the apical membrane of the epithelial cells drives deformation of the tissue, folding it to generate new branches [8]. Coordinated tension of the actomyosin network is essential for generating the cellular forces that drive lumen morphogenesis.

Actomyosin tension is also responsible for fundamental cell movements that define the earliest stages of development. During C. elegans gastrulation, a specific pair of cells must be internalized by the embryo. Apical actomyosin contractility is coordinated by the enveloping cells to bend the embryo and internalize the specified cells. Disrupting myosin activity prevents these movements from occurring [45]. In the ascidian embryo, Rho signaling regulates actomyosin tension at the apical and basolateral surfaces and drives gastrulation by mediating 
other changes in cell shape. Initially, apical constriction induces involution of the endodermal cells. Once complete, actomyosin contractility begins at the basolateral surface, which retracts the cells and envelops them in the center of the embryo [46]. Apical constriction of epithelial cells is thus a fundamental mechanical process driven by actomyosin contractility and essential for several examples of morphogenesis.

\subsection{Regulation of cytoskeletal tension}

Regions of actomyosin contractility can be patterned by conserved signaling networks. In the Drosophila proventriculus, a region of the fly gut, Notch signaling defines the cells that will undergo constriction and invaginate during intestinal organogenesis [47]. In the Drosophila eye, coordination of Hedgehog, Decapentaplegic, and epidermal growth factor signaling induces invagination and furrow formation by the eye disk epithelium [48]. During development of the Drosophila salivary gland, the Forkhead transcription factor regulates actomyosin contractility and apical constriction [10]. Similarly, the transcription factors Snail and Twist regulate contraction and stabilization of the actomyosin network during Drosophila gastrulation, coordinating a ratchet-like mechanism that induces apical constriction [44]. Transcriptional networks are thus essential for regulating the generation of morphogenetic forces throughout development.

Regions of constriction within a tissue can also be patterned by other signaling pathways, including those downstream of Rho GTPases [49]. Spatial restriction of Rho signaling can generate regions of intracellular actomyosin contraction. RhoGEF2, an activator of Rho, stabilizes and drives myosin activity and cytoskeletal tension at the apical surface of gastrulating 
ventral furrow cells in Drosophila, leading to apical constriction and thereby bending the epithelium [50, 51]. Similarly, apical localization of Rho-associated protein kinase (ROCK), a Rho effector, locally increases actomyosin contractility to drive apical constriction in the chick neuroepithelium [52]. Regulating the intracellular localization of contractility can be used to coordinate other types of tissue morphogenesis. PCP can pattern the localization of RhoGEF2, which can establish lateral domains of actomyosin contraction essential for the cellular rearrangements that drive extension of the Drosophila germ band [53].

Actomyosin contractility can also be induced by Rap, a Rho-independent signaling pathway. During Drosophila dorsal closure, PDZ-GEF activates Rap1 and drives constriction at lateral surfaces [54] (Figure 2B). At the earliest stages of Drosophila gastrulation, Rap1 helps localize canoe to adherens junctions, where it mediates linkage to the cytoskeleton and facilitates apical constriction to form the ventral furrow [55]. In Drosophila, Src42 is also essential for extension of the tracheal system. Loss of Src42 leads to shorter and wider tubes within the tracheal network [56]. Signaling through c-Jun N-terminal kinase (JNK) regulates the propagation of physical forces during Drosophila dorsal closure and border cell migration. Activation of JNK leads to upregulation of a variety of genes associated with the actin cytoskeleton and adhesions [57]. JNK signaling can also regulate adhesive contacts in other contexts. In the Drosophila egg chamber, JNK signals mediate the adhesive contacts between cells undergoing collective migration [58].

Understanding how signaling pathways guide epithelial morphogenesis is essential for understanding how cells generate and interpret the forces at work during organ development. 
Transcriptional and intracellular regulation of signaling networks pattern the forces that sculpt epithelia. Whereas many signals that regulate these morphogenetic events have been identified, understanding morphogenesis will require a deeper knowledge of how these signals interface with morphogenetic forces.

\subsection{Adhesive contacts between cells and their neighbors}

The cellular rearrangements that facilitate lumen formation and extension require remodeling of adherens junctions (Figure 2C). Lumen formation in the zebrafish gut occurs as a rod of cells rearranges to expose a central lumen. These cellular rearrangements depend on coordinated remodeling of contacts between adjacent epithelial cells [59]. Similar processes occur during the morphogenesis of other epithelia. In the Drosophila germ band, the junctions at contractile borders are preferentially remodeled as the cells reorganize [53, 60]. As they rearrange, the forces between neighboring cells are transmitted through adherens junctions. This can be observed during invagination of the Drosophila ventral furrow, where the forces of apical constriction are mediated by cadherins throughout the epithelium [55, 61]. Adhesions under tension are stabilized at the cell surface by a reduction in cadherin endocytosis [62]. Similarly, tension is transmitted through adherens junctions in the Drosophila thorax, where PCP signaling generates regions of myosin activity and adhesion remodeling [63]. These processes are essential for cell rearrangements that shape the thorax. It will be interesting to determine whether junctional remodeling is a general mechanism important for other examples of morphogenesis.

The transmission of tension through an epithelium is not limited to adherens junctions. Desmosomes, connected to intermediate filaments, transmit morphogenetic forces during 
development of the mouse mammary gland [64]. Desmosomal cadherins are required for development of the mammary lumen and for proper cell sorting in aggregates of mammary tissue. In the migrating mammary gland epithelium, the cadherin contacts are downregulated and instead cells are linked primarily by desmosomes [21]. These studies suggest that intermediate filament networks may transmit morphogenetic forces in other tissues as well.

In addition to contact with neighboring cells, contact with the surrounding ECM is also a key driver of morphogenesis (Figure 2C). The ECM helps regulate morphogenesis and provides mechanical support to developing organs. Fibronectin is a well-characterized ECM protein that induces branching of the mouse salivary gland [65]. Here, fibronectin is assembled into fibrils downstream of ROCK-mediated actin contractility [66]. The mechanical inputs from fibronectin fibril assembly and ROCK activity are also necessary for stimulating proliferation in the salivary gland epithelium. Fibronectin plays a similar role in morphogenesis of the zebrafish heart: contact between cells and their surrounding ECM facilitates assembly of adherens junctions to maintain integrity of the developing epithelium and coordinates migration necessary for heart tube formation [67]. In zebrafish Kupffer's vesicle, adhesion to ECM restricts apical expansion, leading to changes in cell shape that guide lumen morphogenesis [68]. The regulation of the shape of Kupffer's vesicle is essential for establishing left-right asymmetry in the early embryo.

The physical properties of the ECM can also drive cell fate decisions and morphogenesis. Matrix stiffness is sensed in part through integrins. On stiff ECM, integrins cluster, leading to activation of Rho and assembly of focal adhesions [69]. Cells can sense tension at the ECM through integrin contacts and focal adhesions. Tension at focal adhesions is transmitted to the 
cytoskeleton through vinculin, which can be visualized using a Förster resonance energy transfer (FRET)-based tension sensor [70].

During lumen formation, the physical properties of the surrounding ECM can also affect a range of processes during branching morphogenesis. Tissue geometry can pattern sites of branching of mammary epithelia at regions of elevated mechanical stress [71, 72]. Similarly, branching of vascular endothelial cells is regulated by stiffness of the surrounding ECM [73]. Mechanical forces generated within cells were recently found to be essential for remodeling the ECM to promote migration through the surrounding matrix [74]. Regulation of the bonds between integrins and the ECM is required for epithelial migration. During migration of the Drosophila egg chamber epithelium, integrin levels must be reduced at the trailing edge to allow the cells to release their contacts and migrate as a cohesive group [75]. The regulation of these adhesive contacts is essential for the cell movements that characterize lumen morphogenesis.

\subsection{Fluid pressure during lumen morphogenesis}

In addition to the matrix that surrounds the basal surface of the epithelium, the mechanical forces at the apical surface also regulate lumen formation. For example, cells can tune fluid secretion and pressure within a lumen. Fluid secretion is driven by coordinated ion flux and osmotic gradients [76] (Figure 2D). A key driver of fluid secretion in vertebrates is a chloride channel, the cystic fibrosis transmembrane conductance regulator (CFTR), which plays an essential morphogenetic role in the expansion of tubular organs. For example, in the zebrafish Kupffer's vesicle, CFTR drives fluid secretion to inflate the lumen [77]. 
Changes in lumenal fluid pressure can exert changes in the shape of the surrounding epithelial cells. Intestinal activation of zebrafish CFTR leads to dramatic increases in fluid accumulation, which stretches the epithelial cells [78]. Lumen expansion in the otic vesicle, or zebrafish ear, coincides with a reduction in the volume of the surrounding epithelial cells and thinning of the tissue, suggesting that the initial stages of lumen expansion may be driven by secretion of intracellular fluid reserves before initiating trans-epithelial fluid secretion [79]. Altogether, regulated fluid secretion is an important component of lumen and organ morphogenesis.

Similarly, morphogenesis of the brain depends on properly regulated fluid pressure, which increases proliferation and growth [80]. Studies in the zebrafish have revealed that fluid pressure is also essential for opening the ventricular lumen of the brain [81]. Lumen expansion driven by fluid pressure in the brain is in turn resisted by myosin activity in the surrounding neuroepithelial cells [82], and loss of a key myosin regulator prevents inflation of the zebrafish brain. Epithelial cells surrounding the lumen must respond to increased fluid pressure. In the mammalian bladder, for example, increased pressure stretches the epithelium, leading to trafficking of new membrane to the apical surface [83].

Regulated fluid pressure is also important for lung morphogenesis. Lumen pressure can be increased by ligating the trachea, causing fluid to accumulate within the developing lumen. In response to increased fluid pressure, the lung grows in size [84, 85]. At early stages of lung development, tracheal ligation enhances branching morphogenesis, suggesting that fluid pressure also drives key morphogenetic events in the lung [86]. Conversely, reducing lumenal fluid 
pressure reduces the size of the lung [87]. These studies suggest that fluid pressure is a key regulator of lung morphogenesis. Unraveling the relationship between lumen pressure and lung morphogenesis will require the ability to more precisely control lumen pressure.

\section{Sensing and communicating forces}

\subsection{Signaling at adhesions}

Cells are capable of sensing forces through contacts between neighboring cells and the adjacent ECM. In response to increases in ECM stiffness, integrins act as mechanosensors to activate Rac signaling and increase myosin activity [88-90]. In vascular endothelial cells, tension at adherens junctions recruits vinculin, which helps transmit forces across cell-cell contacts [91]. Increased myosin activity increases cytoskeletal tension and exposes a cryptic binding site in talin, which leads to recruitment of vinculin and stabilization of the junction [92-94].

Under tension, cadherin also undergoes conformational changes that recruit $\alpha$ and $\beta$ catenin [95]. In epithelial cells, junctional cadherins are under continuous actomyosin cytoskeletal tension [96]. As these cells are stretched, tension across the adherens junction is translated into conformational changes in $\alpha$ and $\beta$-catenin that link cadherin to the actin cytoskeleton [95, 97] (Figure 2E). Cells often respond to tension across an epithelium by increasing proliferation. Increased tension across cadherin contacts can drive activation of Yap1 and nuclear accumulation of $\beta$-catenin to increase transcription that promotes entry into the cell cycle [98].

The tension through these contacts leads to conformational changes that can be quantified using a genetically encoded tension sensor $[70,97]$. This sensor consists of two fluorophores 
joined by a flexible linker domain; as the tension across the molecule increases, the distance between the fluorophores increases, reducing the fluorescent emission across the FRET pair

(Figure 2E). This sensor has allowed the forces at work across and between cells to be observed in living cells. Whereas this technique has allowed probing forces at work in individual cells, similar tools are needed to probe the forces at work in multicellular tissues and developing organs.

\subsection{Mechanosensation}

Cells can sense the flow of fluid across their surface. In many organs, lumenal epithelial cells use cilia to sense the shear forces arising from fluid flow (Figure 2F). In the mouse kidney, cilia contain polycystins, which facilitate calcium flux in response to fluid flow. Loss of these calcium channels prevents the sensation of flow and can cause pathological accumulation of fluid within the kidney $[99,100]$. Similarly, cilia have been detected in the mouse heart, where they may sense fluid flow required for cardiac morphogenesis [101].

Detecting fluid flow is also required for the establishment of left-right asymmetry in many species. Two types of cilia contribute to the establishment of left-right asymmetry in mice. Motile cilia in the node drive directional fluid flow, which is detected by sensory cilia. Similar to kidney epithelial cells, these sensory cilia contain Pkd2, a mechanosensitive calcium channel, leading to calcium flux on the left side of the node [102]. Asymmetric calcium flux drives downstream calcium signaling to establish the left side of the body plan, translating a physical process into biochemical signals. 


\subsection{Membrane tension}

Cells are also capable of sensing changes in membrane tension. In response to osmotic stress, cells may increase their volume, which leads to a transient tension at the plasma membrane. This change in volume is detected by a component of the volume-regulated anion channel, SWELL1 (Figure 2F). In response to osmotic stress, SWELL1 mediates anion flux to help normalize cell volume [103].

Piezo proteins are a recently discovered class of mechanosensitive ion channel, and have been found to mediate calcium flux in response to membrane tension in a variety of contexts (Figure 2F). The channels were initially described for their role in the activation of mechanosensitive neurons [104, 105]. Additionally, Piezo proteins have been identified for their roles in the regulation of cell extrusion from epithelia in response to changes in membrane tension [106]. The channels have also been implicated in coordinating morphogenesis of the developing vasculature [107]. It will be interesting to determine how broadly the mechanosensitive Piezo proteins function in the detection of epithelial membrane tension in other systems.

Understanding how cells detect forces will be essential for determining how cells interpret and respond to the physical processes that surround them. Important regulators of mechanosensation have recently been uncovered. Investigating the function of these channels during morphogenesis may provide new insight into the integration between physical forces and the biochemical signaling pathways already known to guide lumen morphogenesis. 


\section{Concluding Remarks}

At its core, morphogenesis is characterized by mechanical interactions that reshape tissues. Many of these global forces are generated locally by the coordinated action of the tissue's constituent cells. A principal driver of these forces is actomyosin contractility, which is responsible for initiating changes in cell shape that translate to morphogenesis across an organ. These forces are transmitted through adhesive contacts between cells and the surrounding ECM to help drive movements that promote bending and extension of the tissue and its internal lumen.

While genetic studies have identified many biochemical signaling pathways, understanding the role of forces has lagged in most morphogenetic systems. To more precisely examine these forces during morphogenesis, we need new tools capable of observing forces at work in whole tissues. To understand the role these forces play during morphogenesis will require the ability to modulate native forces in the developing embryo. Together, tools that allow observation and manipulation of developmental forces will provide a more fundamental understanding of the forces at work during lumen morphogenesis.

Whereas cells are capable of dynamic responses to mechanical forces, the identity of these mechanosensors has remained unclear. Recent work has identified molecules that sense membrane tension, including candidates for the volume-regulated anion channel and stretchactivated cation channels. It will be exciting to learn how these mechanosensors function during lumen morphogenesis.

Altogether, it is clear that mechanical forces are a key driver of lumen formation. The coordination of these forces and biochemical signaling networks governs forms at scales ranging 
from cells to organs to the entire body. To more completely understand lumen morphogenesis, it will be essential to determine how the local forces generated within cells cooperate to generate the long-range interactions that shape epithelia. 


\section{Acknowledgements}

This work was supported in part by grants from the NIH (HL110335, HL118532, HL120142, and CA187692), the NSF (CMMI-1435853), the David \& Lucile Packard Foundation, and the Camille \& Henry Dreyfus Foundation. C.M.N. holds a Career Award at the Scientific Interface from the Burroughs Wellcome Fund.

\section{References}

[1] Hogan BLM, Kolodziej PA. Organogenesis: molecular mechanisms of tubulogenesis. Nat Rev Genet 2002;3:513-23.

[2] Lubarsky B, Krasnow MA. Tube morphogenesis: making and shaping biological tubes. Cell 2003;112:19-28.

[3] Sigurbjörnsdóttir S, Mathew R, Leptin M. Molecular mechanisms of de novo lumen formation. Nat Rev Mol Cell Biol 2014;15:665-76.

[4] Schoenwolf GC, Franks MV. Quantitative analyses of changes in cell shapes during bending of the avian neural plate. Dev Biol 1984;105:257-72.

[5] Keller RE. An experimental analysis of the role of bottle cells and the deep marginal zone in gastrulation of Xenopus laevis. J Exp Zool 1981;216:81-101.

[6] Parks S, Wieschaus E. The Drosophila gastrulation gene concertina encodes a G alpha-like protein. Cell 1991;64:447-58.

[7] Leptin M, Grunewald B. Cell shape changes during gastrulation in Drosophila. Development 1990;110:73-84.

[8] Kim HY, Varner VD, Nelson CM. Apical constriction initiates new bud formation during monopodial branching of the embryonic chicken lung. Development 2013;140:3146-55.

[9] Kadzik RS, Cohen ED, Morley MP, Stewart KM, Lu MM, Morrisey EE. Wnt ligand/Frizzled 2 receptor signaling regulates tube shape and branch-point formation in the lung through control of epithelial cell shape. Proc Natl Acad Sci U S A 2014;111:12444-9.

[10] Myat MM, Andrew DJ. Fork head prevents apoptosis and promotes cell shape change during formation of the Drosophila salivary glands. Development 2000;127:4217-26. 
[11] Monier B, Gettings M, Gay G, Mangeat T, Schott S, Guarner A, et al. Apico-basal forces exerted by apoptotic cells drive epithelium folding. Nature 2015;518:245-8.

[12] Yamaguchi Y, Shinotsuka N, Nonomura K, Takemoto K, Kuida K, Yosida H, et al. Live imaging of apoptosis in a novel transgenic mouse highlights its role in neural tube closure. J Cell Biol 2011;195:1047-60.

[13] Sutherland D, Samakovlis C, Krasnow MA. branchless encodes a Drosophila FGF homolog that controls tracheal cell migration and the pattern of branching. Cell 1996;87:1091-101.

[14] Ghabrial AS, Krasnow MA. Social interactions among epithelial cells during tracheal branching morphogenesis. Nature 2006;441:746-9.

[15] Caussinus E, Colombelli J, Affolter M. Tip-cell migration controls stalk-cell intercalation during Drosophila tracheal tube elongation. Curr Biol 2008;18:1727-34.

[16] Nasevicius A, Larson J, Ekker SC. Distinct requirements for zebrafish angiogenesis revealed by a VEGF-A morphant. Yeast 2000;17:294-301.

[17] Gerhardt H, Golding M, Fruttiger M, Ruhrberg C, Lundkvist A, Abramsson A, et al. VEGF guides angiogenic sprouting utilizing endothelial tip cell filopodia. J Cell Biol 2003;161:116377.

[18] Torres-Vázquez J, Gitler AD, Fraser SD, Berk JD, Pham VN, Fishman MC, et al. Semaphorin-plexin signaling guides patterning of the developing vasculature. Dev Cell 2004; 7:117-23.

[19] Vasilyev A, Liu Y, Mudumana S, Mangos S, Lam P-y, Majumdar A, et al. Collective Cell Migration Drives Morphogenesis of the Kidney Nephron. PLoS Biol 2009; 7:e1000009.

[20] Ewald AJ, Brenot A, Duong M, Chan BS, Werb Z. Collective epithelial migration and cell rearrangements drive mammary branching morphogenesis. Dev Cell 2008;14:570-81.

[21] Ewald AJ, Huebner RJ, Palsdottir H, Lee JK, Perez MJ, Jorgens DM, et al. Mammary collective cell migration involves transient loss of epithelial features and individual cell migration within the epithelium. J Cell Sci 2012;125:2638-54.

[22] Nguyen-Ngoc K-V, Cheung KJ, Brenot A, Shamir ER, Gray RS, Hines WC, et al. ECM microenvironment regulates collective migration and local dissemination in normal and malignant mammary epithelium. Proc Natl Acad Sci U S A 2012;109:E2595-604.

[23] Irvine KD, Wieschaus E. Cell intercalation during Drosophila germband extension and its regulation by pair-rule segmentation genes. Development 1994;120:827-41. 
[24] Rauzi M, Lenne P-F, Lecuit T. Planar polarized actomyosin contractile flows control epithelial junction remodelling. Nature 2010;468:1110-4.

[25] Fernandez-Gonzalez R, Zallen JA. Oscillatory behaviors and hierarchical assembly of contractile structures in intercalating cells. Phys Biol 2011;8:045005.

[26] Sawyer JK, Choi W, Jung K-C, He L, Harris NJ, Peifer M. A contractile actomyosin network linked to adherens junctions by Canoe/afadin helps drive convergent extension. Mol Biol Cell 2011;22:2491-508.

[27] Iwaki DD, Johansen KA, Singer JB, Lengyel JA. drumstick, bowl, and lines are required for patterning and cell rearrangement in the Drosophila embryonic hindgut. Dev Biol 2001;240:61126.

[28] Park M, Moon RT. The planar cell-polarity gene stbm regulates cell behaviour and cell fate in vertebrate embryos. Nat Cell Biol 2002;4:20-5.

[29] Lienkamp SS, Liu K, Karner CM, Carroll TJ, Ronneberger O, Wallingford JB, et al. Vertebrate kidney tubules elongate using a planar cell polarity-dependent, rosette-based mechanism of convergent extension. Nat Genet 2012;44:1382-7.

[30] Skoglund P, Rolo A, Chen X, Gumbiner BM, Keller R. Convergence and extension at gastrulation require a myosin IIB-dependent cortical actin network. Development 2008;135:2435-44.

[31] Kibar Z, Vogan KJ, Groulx N, Justice MJ, Underhill DA, Gros P. Ltap, a mammalian homolog of Drosophila Strabismus/Van Gogh, is altered in the mouse neural tube mutant Looptail. Nat Genet 2001;28:251-5.

[32] Nishimura T, Honda H, Takeichi M. Planar cell polarity links axes of spatial dynamics in neural-tube closure. Cell 2012;149:1084-97.

[33] Townes PL, Holtfreter J. Directed movements and selective adhesion of embryonic amphibian cells. J Exp Zool 1955;128:53-120.

[34] Steinberg MS. On the mechanism of tissue reconstruction by dissociated cells. I. Population kinetics, differential adhesiveness. and the absence of directed migration. Proc Natl Acad Sci U S A 1962;48:1577-82.

[35] Davis GS, Phillips HM, Steinberg MS. Germ-layer surface tensions and \&quot;tissue affinities\&quot; in Rana pipiens gastrulae: quantitative measurements. Dev Biol 1997;192:63044. 
[36] Foty RA, Steinberg MS. The differential adhesion hypothesis: a direct evaluation. Dev Biol 2005;278:255-63.

[37] Krieg M, Arboleda-Estudillo Y, Puech P-H, Käfer J, Graner F, Müller DJ, et al. Tensile forces govern germ-layer organization in zebrafish. Nat Cell Biol 2008;10:429-36.

[38] Zhou J, Kim HY, Davidson LA. Actomyosin stiffens the vertebrate embryo during crucial stages of elongation and neural tube closure. Development 2009;136:677-88.

[39] Maître J-L, Berthoumieux H, Krens SFG, Salbreux G, Jülicher F, Paluch E, et al. Adhesion functions in cell sorting by mechanically coupling the cortices of adhering cells. Science 2012;338:253-6.

[40] Maître J-L, Niwayama R, Turlier H, Nédélec F, Hiiragi T. Pulsatile cell-autonomous contractility drives compaction in the mouse embryo. Nat Cell Biol 2015.

[41] Cerchiari AE, Garbe JC, Jee NY, Todhunter ME, Broaders KE, Peehl DM, et al. A strategy for tissue self-organization that is robust to cellular heterogeneity and plasticity. Proc Natl Acad Sci U S A 2015;112:2287-92.

[42] Franke JD, Montague RA, Kiehart DP. Nonmuscle myosin II generates forces that transmit tension and drive contraction in multiple tissues during dorsal closure. Curr Biol 2005;15:220821.

[43] Solon J, Kaya-Çopur A, Colombelli J, Brunner D. Pulsed forces timed by a ratchet-like mechanism drive directed tissue movement during dorsal closure. Cell 2009;137:1331-42.

[44] Martin AC, Kaschube M, Wieschaus EF. Pulsed contractions of an actin-myosin network drive apical constriction. Nature 2009;457:495-9.

[45] Lee J, Goldstein B. Mechanisms of cell positioning during C. elegans gastrulation. Development 2003.

[46] Sherrard K, Robin F, Lemaire P, Munro E. Sequential activation of apical and basolateral contractility drives ascidian endoderm invagination. Curr Biol 2010;20:1499-510.

[47] Fuss B, Josten F, Feix M, Hoch M. Cell movements controlled by the Notch signalling cascade during foregut development in Drosophila. Development 2004;131:1587-95.

[48] Escudero LM, Bischoff M, Freeman M. Myosin II regulates complex cellular arrangement and epithelial architecture in Drosophila. Dev Cell 2007;13:717-29.

[49] Hall A. Rho GTPases and the actin cytoskeleton. Science 1998;279:509-14. 
[50] Fox DT, Peifer M. Abelson kinase (Abl) and RhoGEF2 regulate actin organization during cell constriction in Drosophila. Development 2007;134:567-78.

[51] Barrett K, Leptin M, Settleman J. The Rho GTPase and a putative RhoGEF mediate a signaling pathway for the cell shape changes in Drosophila gastrulation. Cell 1997;91:905-15.

[52] Nishimura T, Takeichi M. Shroom3-mediated recruitment of Rho kinases to the apical cell junctions regulates epithelial and neuroepithelial planar remodeling. Development 2008;135:1493-502.

[53] Levayer R, Pelissier-Monier A, Lecuit T. Spatial regulation of Dia and Myosin-II by RhoGEF2 controls initiation of E-cadherin endocytosis during epithelial morphogenesis. Nat Cell Biol 2011;13:529-40.

[54] Boettner B, Van Aelst L. The Rap GTPase activator Drosophila PDZ-GEF regulates cell shape in epithelial migration and morphogenesis. Mol Cell Biol 2007;27:7966-80.

[55] Sawyer JK, Harris NJ, Slep KC, Gaul U, Peifer M. The Drosophila afadin homologue Canoe regulates linkage of the actin cytoskeleton to adherens junctions during apical constriction. J Cell Biol 2009;186:57-73.

[56] Nelson KS, Khan Z, Molnár I, Mihály J, Kaschube M, Beitel GJ. Drosophila Src regulates anisotropic apical surface growth to control epithelial tube size. Nat Cell Biol 2012;14:518-25.

[57] Jasper H, Benes V, Schwager C, Sauer S, Clauder-Münster S, Ansorge W, et al. The genomic response of the Drosophila embryo to JNK signaling. Dev Cell 2001;1:579-86.

[58] Llense F, Martín-Blanco E. JNK signaling controls border cell cluster integrity and collective cell migration. Curr Biol 2008;18:538-44.

[59] Alvers AL, Ryan S, Scherz PJ, Huisken J, Bagnat M. Single continuous lumen formation in the zebrafish gut is mediated by smoothened-dependent tissue remodeling. Development 2014;141:1110-9.

[60] Bertet C, Sulak L, Lecuit T. Myosin-dependent junction remodelling controls planar cell intercalation and axis elongation. Nature 2004;429:667-71.

[61] Martin AC, Gelbart M, Fernandez-Gonzalez R, Kaschube M, Wieschaus EF. Integration of contractile forces during tissue invagination. J Cell Biol 2010;188:735-49.

[62] Izumi G, Sakisaka T, Baba T, Tanaka S, Morimoto K, Takai Y. Endocytosis of E-cadherin regulated by Rac and Cdc42 small G proteins through IQGAP1 and actin filaments. J Cell Biol 2004;166:237-48. 
[63] Bosveld F, Bonnet I, Guirao B, Tlili S, Wang Z, Petitalot A, et al. Mechanical control of morphogenesis by Fat/Dachsous/Four-jointed planar cell polarity pathway. Science 2012;336:724-7.

[64] Runswick SK, O\&apos;Hare MJ, Jones L, Streuli CH, Garrod DR. Desmosomal adhesion regulates epithelial morphogenesis and cell positioning. Nat Cell Biol 2001;3:823-30.

[65] Sakai T, Larsen M, Yamada KM. Fibronectin requirement in branching morphogenesis. Nature 2003;423:876-81.

[66] Daley WP, Gulfo KM, Sequeira SJ, Larsen M. Identification of a mechanochemical checkpoint and negative feedback loop regulating branching morphogenesis. Dev Biol 2009;336:169-82.

[67] Trinh LA, Stainier DYR. Fibronectin regulates epithelial organization during myocardial migration in zebrafish. Dev Cell 2004;6:371-82.

[68] Compagnon J, Barone V, Rajshekar S, Kottmeier R, Pranjic-Ferscha K, Behrndt M, et al. The notochord breaks bilateral symmetry by controlling cell shapes in the zebrafish laterality organ. Dev Cell 2014;31:774-83.

[69] Paszek MJ, Zahir N, Johnson KR, Lakins JN, Rozenberg GI, Gefen A, et al. Tensional homeostasis and the malignant phenotype. Cancer Cell 2005;8:241-54.

[70] Grashoff C, Hoffman BD, Brenner MD, Zhou R, Parsons M, Yang MT, et al. Measuring mechanical tension across vinculin reveals regulation of focal adhesion dynamics. Nature 2010;466:263-6.

[71] Nelson CM, VanDuijn MM, Inman JL, Fletcher DA, Bissell MJ. Tissue Geometry Determines Sites of Mammary Branching Morphogenesis in Organotypic Cultures. Science (New York, NY) 2006;314:298-300.

[72] Gjorevski N, Nelson CM. Endogenous patterns of mechanical stress are required for branching morphogenesis. Integr Biol (Camb) 2010;2:424-34.

[73] Myers KA, Applegate KT, Danuser G, Fischer RS, Waterman CM. Distinct ECM mechanosensing pathways regulate microtubule dynamics to control endothelial cell branching morphogenesis. J Cell Biol 2011;192:321-34.

[74] Gjorevski N, Piotrowski AS, Varner VD, Nelson CM. Dynamic tensile forces drive collective cell migration through three-dimensional extracellular matrices. Sci Rep 2015;5:11458. 
[75] Lewellyn L, Cetera M, Horne-Badovinac S. Misshapen decreases integrin levels to promote epithelial motility and planar polarity in Drosophila. J Cell Biol 2013;200:721-9.

[76] Barrett KE, Keely SJ. Chloride secretion by the intestinal epithelium: molecular basis and regulatory aspects. Annu Rev Physiol 2000;62:535-72.

[77] Navis A, Marjoram L, Bagnat M. Cftr controls lumen expansion and function of Kupffer's vesicle in zebrafish. Development 2013;140:1703-12.

[78] Bagnat M, Navis A, Herbstreith S, Brand-Arzamendi K, Curado S, Gabriel S, et al. Cse11 is a negative regulator of CFTR-dependent fluid secretion. Curr Biol 2010;20:1840-5.

[79] Hoijman E, Rubbini D, Colombelli J, Alsina B. Mitotic cell rounding and epithelial thinning regulate lumen growth and shape. Nature communications 2015;6:7355.

[80] Desmond ME, Jacobson AG. Embryonic brain enlargement requires cerebrospinal fluid pressure. Dev Biol 1977;57:188-98.

[81] Lowery LA, Sive H. Initial formation of zebrafish brain ventricles occurs independently of circulation and requires the nagie oko and snakehead/atp1a1a.1 gene products. Development 2005;132:2057-67.

[82] Gutzman JH, Sive H. Epithelial relaxation mediated by the myosin phosphatase regulator Mypt1 is required for brain ventricle lumen expansion and hindbrain morphogenesis. Development 2010;137:795-804.

[83] Wang E, Truschel S, Apodaca G. Analysis of hydrostatic pressure-induced changes in umbrella cell surface area. Methods 2003;30:207-17.

[84] Hashim E, Laberge JM, Chen MF, Quillen EW. Reversible tracheal obstruction in the fetal sheep: effects on tracheal fluid pressure and lung growth. J Pediatr Surg 1995;30:1172-7.

[85] Nardo L, Hooper SB, Harding R. Stimulation of lung growth by tracheal obstruction in fetal sheep: relation to luminal pressure and lung liquid volume. Pediatr Res 1998;43:184-90.

[86] Unbekandt M, del Moral P-M, Sala FG, Bellusci S, Warburton D, Fleury V. Tracheal occlusion increases the rate of epithelial branching of embryonic mouse lung via the FGF10FGFR2b-Sprouty2 pathway. Mech Dev 2008;125:314-24.

[87] Alcorn D, Adamson TM, Lambert TF, Maloney JE, Ritchie BC, Robinson PM. Morphological effects of chronic tracheal ligation and drainage in the fetal lamb lung. J Anat 1977;123:649-60. 
[88] Riveline D, Zamir E, Balaban NQ, Schwarz US, Ishizaki T, Narumiya S, et al. Focal contacts as mechanosensors: externally applied local mechanical force induces growth of focal contacts by an mDia1-dependent and ROCK-independent mechanism. J Cell Biol 2001; 153:1175-86.

[89] Pasapera AM, Plotnikov SV, Fischer RS, Case LB, Egelhoff TT, Waterman CM. Rac1Dependent Phosphorylation and Focal Adhesion Recruitment of Myosin IIA Regulates Migration and Mechanosensing. Curr Biol 2014;25:175-86.

[90] Schiller HB, Hermann M-R, Polleux J, Vignaud T, Zanivan S, Friedel CC, et al. $\beta 1$ - and $\alpha$ class integrins cooperate to regulate myosin II during rigidity sensing of fibronectin-based microenvironments. Nat Cell Biol 2013;15:625-36.

[91] Huveneers S, Oldenburg J, Spanjaard E, van der Krogt G, Grigoriev I, Akhmanova A, et al. Vinculin associates with endothelial VE-cadherin junctions to control force-dependent remodeling. J Cell Biol 2012;196:641-52.

[92] del Rio A, Perez-Jimenez R, Liu R, Roca-Cusachs P, Fernandez JM, Sheetz MP. Stretching single talin rod molecules activates vinculin binding. Science 2009;323:638-41.

[93] Pasapera AM, Schneider IC, Rericha E, Schlaepfer DD, Waterman CM. Myosin II activity regulates vinculin recruitment to focal adhesions through FAK-mediated paxillin phosphorylation. J Cell Biol 2010;188:877-90.

[94] Barry AK, Wang N, Leckband DE. Local VE-cadherin mechanotransduction triggers longranged remodeling of endothelial monolayers. J Cell Sci 2015;128:1341-51.

[95] Drees F, Pokutta S, Yamada S, Nelson WJ, Weis WI. Alpha-catenin is a molecular switch that binds E-cadherin-beta-catenin and regulates actin-filament assembly. Cell 2005;123:903-15.

[96] Borghi N, Sorokina M, Shcherbakova OG, Weis WI, Pruitt BL, Nelson WJ, et al. Ecadherin is under constitutive actomyosin-generated tension that is increased at cell-cell contacts upon externally applied stretch. Proc Natl Acad Sci U S A 2012;109:12568-73.

[97] Buckley CD, Tan J, Anderson KL, Hanein D, Volkmann N, Weis WI, et al. The minimal cadherin-catenin complex binds to actin filaments under force. Science 2014;346:1254211-.

[98] Benham-Pyle BW, Pruitt BL, Nelson WJ. Cell adhesion. Mechanical strain induces Ecadherin-dependent Yap1 and $\beta$-catenin activation to drive cell cycle entry. Science 2015;348:1024-7.

[99] Nauli SM, Alenghat FJ, Luo Y, Williams E, Vassilev P, Li X, et al. Polycystins 1 and 2 mediate mechanosensation in the primary cilium of kidney cells. Nat Genet 2003;33:129-37. 
[100] Nauli SM, Kawanabe Y, Kaminski JJ, Pearce WJ, Ingber DE, Zhou J. Endothelial cilia are fluid shear sensors that regulate calcium signaling and nitric oxide production through polycystin-1. Circulation 2008;117:1161-71.

[101] Slough J, Cooney L, Brueckner M. Monocilia in the embryonic mouse heart suggest a direct role for cilia in cardiac morphogenesis. Dev Dyn 2008;237:2304-14.

[102] Yoshiba S, Shiratori H, Kuo IY, Kawasumi A, Shinohara K, Nonaka S, et al. Cilia at the node of mouse embryos sense fluid flow for left-right determination via Pkd2. Science 2012;338:226-31.

[103] Qiu Z, Dubin AE, Mathur J, Tu B, Reddy K, Miraglia LJ, et al. SWELL1, a plasma membrane protein, is an essential component of volume-regulated anion channel. Cell 2014; $157: 447-58$.

[104] Ranade SS, Woo S-H, Dubin AE, Moshourab RA, Wetzel C, Petrus M, et al. Piezo2 is the major transducer of mechanical forces for touch sensation in mice. Nature 2014;516:121-5.

[105] Coste B, Mathur J, Schmidt M, Earley TJ, Ranade S, Petrus MJ, et al. Piezo1 and Piezo2 are essential components of distinct mechanically activated cation channels. Science 2010;330:55-60.

[106] Eisenhoffer GT, Loftus PD, Yoshigi M, Otsuna H, Chien C-B, Morcos PA, et al. Crowding induces live cell extrusion to maintain homeostatic cell numbers in epithelia. Nature 2012;484:546-9.

[107] Ranade SS, Qiu Z, Woo S-H, Hur SS, Murthy SE, Cahalan SM, et al. Piezo1, a mechanically activated ion channel, is required for vascular development in mice. Proc Natl Acad Sci U S A 2014;111:10347-52. 


\section{Figure Legends}

Figure 1: Mechanical interactions that shape epithelia. (A) Schematic of epithelial bending during morphogenesis of the neural tube and lung in chicken embryos. (B) At the cellular level, bending can be initiated by constriction of the apical surface of epithelial cells. This local change in cell shape drives global deformation of the epithelial tissue. (C) Schematic representation of collective migration characteristic of lumen outgrowth in the vertebrate vasculature and Drosophila trachea. (D) Convergent extension is coordinated by cellular rearrangements between neighboring epithelial cells. Coordination of these rearrangements allows cells to compact in one direction and extend in another. (E) Diagram of cell sorting events. Cortical tension is capable of segregating tissue layers; binding to ECM can direct the proper orientation of these layers.

Figure 2: Cellular mechanisms of force generation. (A) During apical constriction, myosin motors generate contractile forces in the actin cytoskeleton at the apical surface of epithelial cells. (B) Diagram of key signaling pathways: Rho, ROCK, and Rap regulate actomyosin contractility. (C) Schematic representation of the intercellular contacts that sense and transmit forces between cells and the surrounding ECM. Tight and adherens junctions provide linkage between neighboring cells and the actin cytoskeleton. Desmosomes link neighboring cells and the intermediate filament network. Integrins mediate linkage between the actin cytoskeleton and the surrounding ECM. (D) Schematic representation of the ion channels and currents that regulate epithelial fluid secretion. (E) Schematic representation of the role of tension stabilizing the interaction between $\alpha$ - and $\beta$-catenin to drive downstream signaling events. A diagram of 
FRET demonstrates the two fluorophores connected by a flexible linker domain; the fluorescent energy transferred between them is determined by the distance between the fluorophores. Stretching of native vinculin under tension allows recruitment of talin, which can drive downstream signaling. (F) Examples of several cation channels responsible for detecting membrane tension in response to shear stress, fluid flow, and osmotic pressure. 


\section{Figure 1}
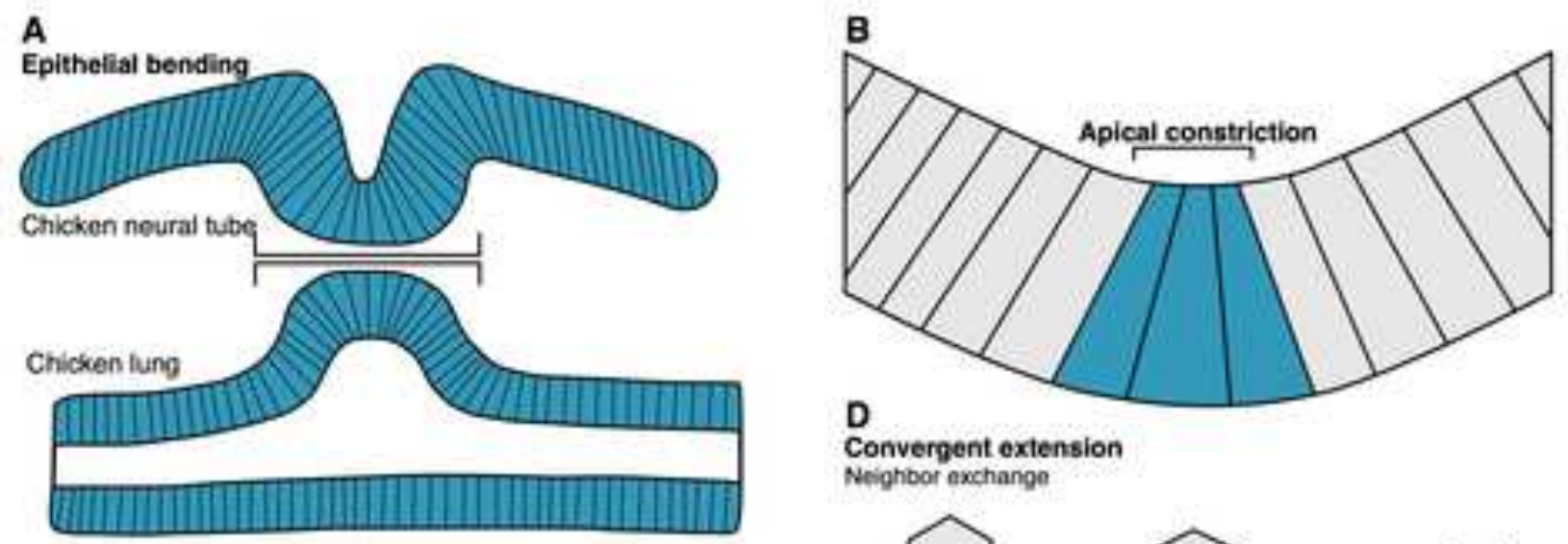

\section{D \\ Convergent extension} Nesighbor exchange
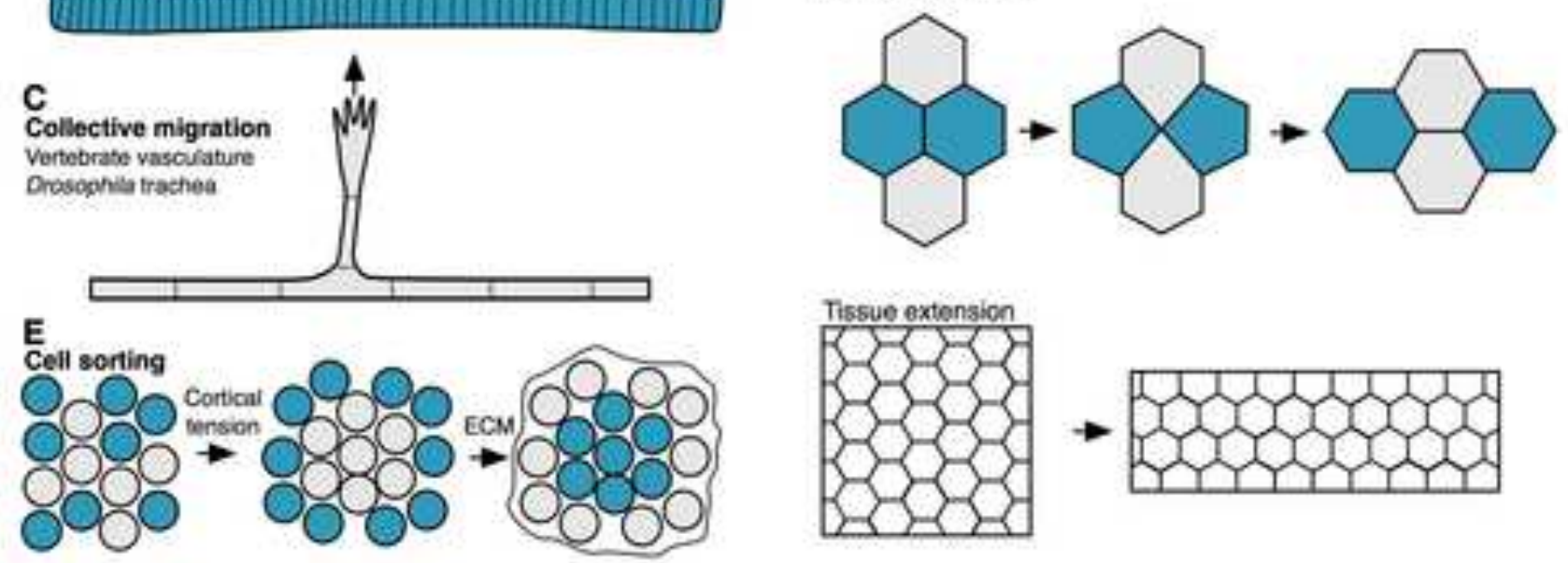
Figure 2
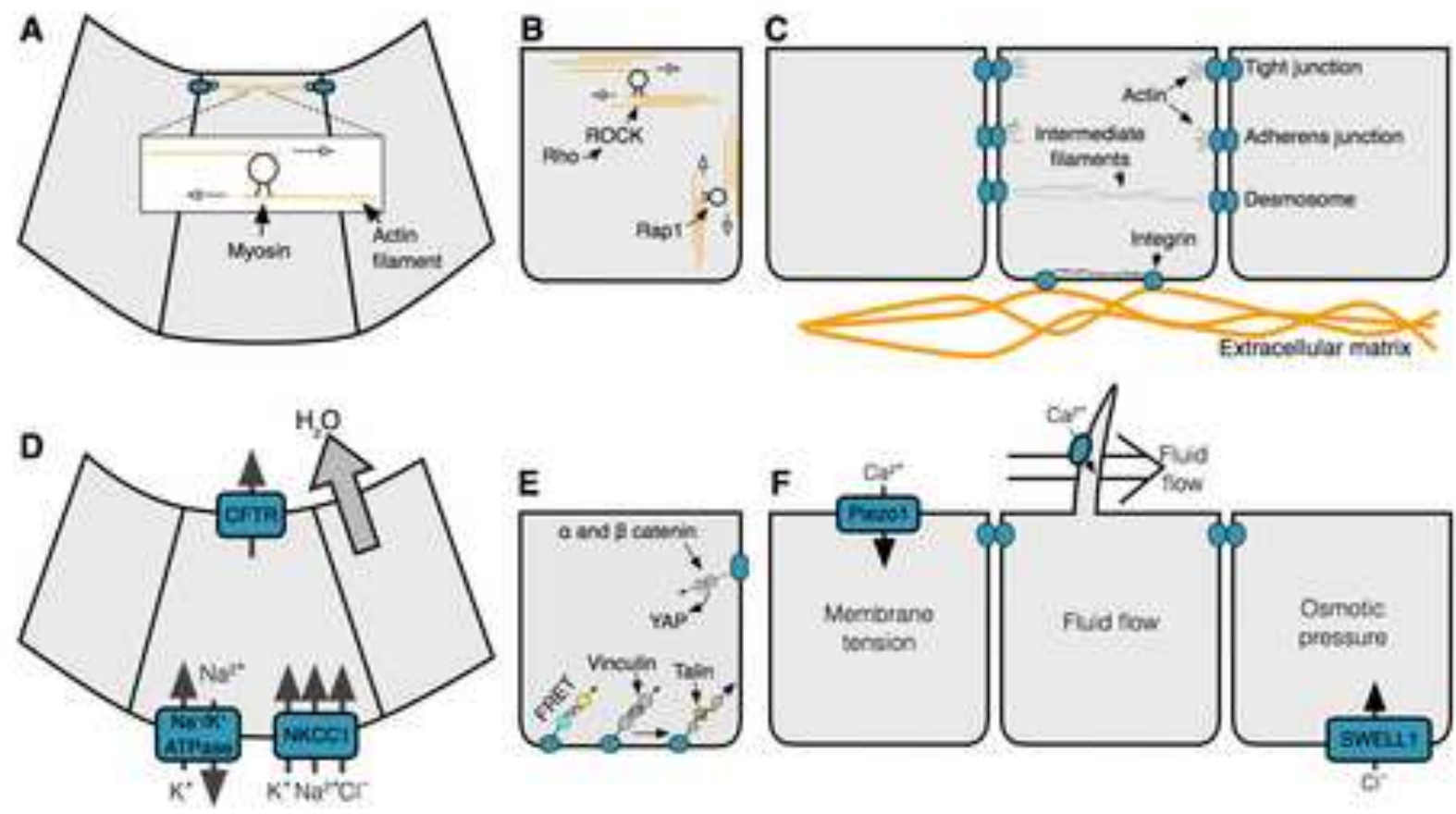